\title{
A comparative analysis of serial and free recall
}

\author{
KRYSTAL A. KLEIN, KELLY M. ADDIS, and MICHAEL J. KAHANA \\ Brandeis University, Waltham, Massachusetts
}

\begin{abstract}
Multitrial free and serial recall tasks differ both in recall instruction and in presentation order across trials. Waugh (1961) compared these paradigms with an intermediate condition: free recall with constant presentation order. She concluded that differences between free and serial recall were due only to recall instructions, and not to presentation order. The present study reevaluated the relation between free and serial recall, using Waugh's three conditions. By examining recall transitions and the organization of information retained across trials, we conclude that presentation order is an important factor, causing participants to exhibit the same temporal associations in serial recall and in free recall with constant presentation order.
\end{abstract}

The present study represents an attempt to reconcile findings from two classic learning paradigms: serial learning and multitrial free recall. In serial learning, participants must recall a list in its presented order, and this order is preserved from trial to trial. In multitrial free recall, participants may recall list items in any order, and the presentation order is randomized from trial to trial. Over the course of multiple study-test trials, participants' overall recall level increases in both tasks, thus demonstrating transfer from earlier to later trials.

Historically, learning across trials has been a topic of great interest in both free and serial recall paradigms (e.g., Hull et al., 1940; Johnson, 1991; L. B. Ward, 1937). Considerable current attention, however, has been given instead to understanding the mechanisms underlying singletrial variants of free and serial recall (e.g., Anderson, Bothell, Lebiere, \& Matessa, 1998; Botvinick \& Plaut, 2004; Brown, Preece, \& Hulme, 2000; Cowan, Baddeley, Elliott, \& Norris, 2003; Farrell \& Lewandowsky, 2002; Howard \& Kahana, 2002; Nairne, Neath, Serra, \& Byun, 1997; for exceptions, see Becker \& Lim, 2003, and Raaijmakers, 2003). Moreover, although the vast majority of studies of free and serial recall have focused on understanding performance within each paradigm individually, our aim is to expose the relation between these paradigms. This aim derives from the observation of similar trends across these two tasks, which, in aggregate, suggest that free and serial recall share common associative mechanisms (Hulme, Stuart, Brown, \& Morin, 2003; Kahana \& Caplan, 2002; G. Ward, Woodward, Stevens, $\&$ Stinson, 2003).

The first two authors contributed equally to this research, and the order of these two authors was chosen randomly. The authors acknowledge support from National Institutes of Health Grant MH55687. We thank Geoff Ward, Marc Howard, Arne Ekstrom, and Per Sederberg for helpful comments on the manuscript. Correspondence concerning this article should be addressed to M. J. Kahana, Department of Psychology, University of Pennsylvania, Suite 302C, 3401 Walnut Street, Philadelphia, PA 19107 (e-mail: kahana@sas.upenn.edu).
How can one quantitatively measure and account for the differences between serial and free recall? Do the two conditions have enough in common for comparison? Conceptually, serial and free recall tasks vary only in that serial recall requires a participant to learn order information (Drewnowski \& Murdock, 1980). Multitrial versions of these two tasks also vary in that free recall lists are traditionally presented in different orders with each successive trial, whereas serial recall lists remain in constant order over all trials.

Waugh (1961) set out to determine whether the differences between performance in multitrial free recall and serial learning could be attributed to recall instructions or to differences in the order of presentation of the study list across trials. She compared serial recall with two different free recall conditions: Either the 48-word list to be studied was held in constant order across six trials, as in serial recall (free recall with constant presentation order; FR-constant), or it was shuffled after each of the six trials, as in traditional free recall (free recall with varied presentation order; FR-varied). For each condition, Waugh calculated learning curves, which show how probability of recall increases across trials. Whereas learning curves in the two free recall conditions did not differ, serial recall performance started out at a lower level but, over trials, overlook that of the two free recall conditions. Waugh hypothesized that the difference in rate of learning between free and serial recall resulted from the strategies that were employed, due to the different recall instructions. On the basis of the similarity of the two free recall conditions, Waugh further concluded that "repeated contiguity between the items to be learned is in this instance an unimportant variable" (p. 499).

Given that associative processes play an important role in episodic memory (Kahana, 1996), it is surprising that Waugh (1961) failed to detect an effect of repeated contiguity in her study. Indeed, Jung and Skeebo (1967), who replicated Waugh's free recall conditions, found that FR-constant leads to significantly faster learning than does FR-varied, as would be expected if repeated conti- 
guity plays an important role in free recall. Jung and Skeebo's study had a number of limitations, including the fact that their participants were children who studied just one list each in a between-subjects design; nevertheless, the results are discordant with Waugh's conclusions. If Waugh was premature in concluding that input order across trials was unimportant, further analysis of the FR-constant condition, in comparison with standard multitrial free and serial recall, could provide valuable clues about the differences and similarities between free and serial recall.

In attempting to reevaluate the relation between free and serial recall, it is important to carefully analyze the aspects of performance in each paradigm that suggest common associative mechanisms. An important new tool in the analysis of list memory experiments involves the measurement of response order during recall. In both free and serial recall, after recalling any given item, participants tend to recall another item from a nearby position in the study list. Moreover, these transitions tend to be to later list items, rather than to earlier list items. This occurs both in free recall (Kahana, 1996; G. Ward et al., 2003) and in the pattern of errors made in probed recall following serial learning (Kahana \& Caplan, 2002; Raskin \& Cook, 1937). As in Waugh (1961), in the present study, multitrial serial recall is compared with FR-constant and FR-varied conditions. Our aim was to foster a more detailed and quantitative understanding of the relation between the free and the serial learning paradigms, comparing them not only on classic analyses, such as learning and serial position curves, but also on measures of temporal associative tendencies.

\section{METHOD}

\section{Participants}

Twelve native English speakers between the ages of 18 and 30 years were recruited to participate in a 10 -session memory and learning study for payment.

\section{Design}

The participants were tested in each of three conditions.

1. Free recall with varied presentation order (FR-varied condition). List words appeared in different random orders on each trial, and the participants were instructed to recall the list in any order, without repeating words.

2. Free recall with constant presentation order (FR-constant condition). List words appeared in the same order on each trial, and the participants were instructed to recall the list in any order, without repeating words.

3. Serial recall with constant presentation order (SR condition). List words appeared in the same order on each trial, and the participants were instructed to recall the list in order, without backtracking.

Experimental lists for each participant contained 19 words, randomly selected from a large pool of two-syllable (mean word length of 6.5 letters), high-frequency (mean Kučera-Francis written frequency of 31.9) nouns sampled from the MRC database (Wilson, 1988). Words were not repeated across lists for a given participant, but the same word pool was used to generate each participant's lists. Session 1 was a practice session consisting of one list from each of the experimental conditions described above. Our reason for including a full hour-long practice session was to avoid differences in learning-to-learn effects across the three conditions (Dallett, 1963). Each subsequent session contained a single condition, commencing with one practice list, followed by seven test lists. The participants were always informed about which condition they were going to receive. The nine test sessions were divided into 3 three-session blocks; each block contained one session from each condition. The order of the three conditions was the same across all three blocks (e.g., FR-V, FR-C, SR, FR-V, FR-C, SR, FR-V, FR-C, SR or SR, FR-V, FR-C, SR, FR-V, FR-C, SR, FR-V, FR-C). All six possible permutations of the three conditions were used and were counterbalanced across participants. By excluding data from the practice session (and practice lists from the beginning of each test session), we ensured that the participants were performing at a steady-state level, and we were thus able to analyze the data from all test lists simultaneously, without worry of an effect of session number or other learning-to-learn effects.

\section{Procedure}

The study was carried out in soundproof testing rooms. An experimenter was present during the entire practice session and for the practice lists in all the subsequent sessions. List presentation and recording of responses were controlled by computer. List items were presented auditorily every $1.5 \mathrm{sec}$ (each word in the pool was recorded by a female speaker with clear diction). Following the presentation of the list, the beginning of the vocal recall period was signaled by a row of asterisks appearing on the screen, a 300-msec tone, and the visual instructions to "Please begin recalling the list." After recalling all remembered words, the participant terminated the recall period by pressing the space bar on the keyboard. Spoken responses were recorded to the computer for later scoring. The participants continued this study-test routine for a total of five trials before advancing to the next list. Each session lasted approximately $45 \mathrm{~min}$, and the participants were allowed to take short breaks between lists. The participants took part in no more than 1 session per day and completed all 10 sessions in no more than 2 weeks.

\section{RESULTS}

Because serial recall requires the participant to recall in the order of list presentation, whereas free recall does not, two methods of scoring were considered when the data from the present experiment were analyzed. In item scoring, a recalled item was scored as correct if it had been presented on the current list, without regard to order. In relative order scoring, an item was scored as correct if it was recalled directly following an item from an earlier study position (Drewnowski \& Murdock, 1980). The first recall was always scored as correct in the relative order scoring method. The decision to use relative order scoring, rather than strict positional scoring, was based on the use of oral recall of supraspan length lists. In our experience, instructions to indicate skipped items in recall are both awkward and unreliable, especially after several omissions of items at earlier serial positions. Strict positional scoring would, therefore, underestimate the recall probabilities for later serial positions. Relative order scoring has been shown to be less sensitive than positional scoring to methodological issues of this type (Addis \& Kahana, 2004). In addition, because our main goal was to compare overall learning of list items across the three recall conditions, item scoring provides a fair comparison of recall performance, regardless of recall instructions. 
Figure 1 shows the proportion of the list that was correctly recalled, using the item scoring method as a function of trial number for each of the recall conditions, as well as the proportion of correct-order-scored recall for serial recall. Because very few words were recalled out of order in the SR condition, only item curves will be discussed for this and subsequent analyses.

A 3 (condition) $\times 5$ (trial) repeated measures analysis of variance (ANOVA) was conducted. As was expected, the proportion of the list correctly recalled increased over trials in each of the three conditions, as confirmed by a main effect of trial $\left[F(4,44)=250.0, M S_{\mathrm{e}}=0.006\right.$, $p<.001]$. In addition, the learning curves were negatively accelerated. This was confirmed by a significant quadratic term $\left[F(1,11)=232.6, M S_{\mathrm{e}}=0.002, p<\right.$ $.001]$. The main effect of condition $[F(2,22)=14.4$, $\left.M S_{\mathrm{e}}=0.003, p<.001\right]$ was statistically significant, reflecting the finding that performance in the FR-varied condition was significantly lower overall than performance in either the FR-constant $[t(11)=4.90, p<$ $.001]$ or the $\mathrm{SR}[t(11)=5.1, p<.001]$ condition. The $\mathrm{SR}$ and $\mathrm{FR}$-constant conditions did not differ significantly overall $[t(11)=0.7$, n.s. $]$. Furthermore, the interaction of condition and trial $\left[F(8,88)=22.0, M S_{\mathrm{e}}=\right.$ $0.001, p<.001]$ was statistically significant. Consistent with this significant interaction, we found that fewer items in Trial 1 were recalled in the SR condition than in either FR condition. During later trials, the mean SR curve overtakes both FR curves, and the FR-constant curve overtakes the FR-varied curve.

Figure 2 plots serial position curves for lists in each recall condition for Trials $1-5$. Of particular interest is the comparison between Trials 1 and 2, because this shows the change in recall for the first trial in which the presentation of items differed between FR conditionsthat is, order was randomized in FR-varied, but not in FRconstant. To compare the changes in recall probabilities in the three recall conditions across trials, a 3 (condition) $\times 3$ (serial position bin) $\times 5$ (trial) repeated measures ANOVA was computed, where serial position bins consisted of the primacy region (Serial Positions 1-4), the middle region (Serial Positions 5-15), and the recency region (Serial Positions 16-19). The participants increased their recall over trials, as confirmed by a significant main effect of trial $\left[F(4,44)=298.5, M S_{\mathrm{e}}=\right.$ $0.012, p<.001]$. In addition, main effects of condition $\left[F(2,22)=22.0, M S_{\mathrm{e}}=0.006, p<.001\right]$ and serial position bin $\left[F(2,22)=31.3, M S_{\mathrm{e}}=0.027, p<.001\right]$ were found. A significant interaction of condition and serial position bin $\left[F(4,4)=24.6, M S_{\mathrm{e}}=0.008, p<.001\right]$ indicates that the level of recall in the primacy and recency regions differed across conditions.

Figure 2A shows that in Trial 1, both the FR-constant and the FR-varied conditions exhibit a large recency effect and a moderate primacy effect, replicating the standard free recall serial position curve (Murdock, 1962). The SR condition, in contrast, shows a moderate primacy effect and a small recency effect, replicating the standard serial position curve for serial recall (Drewnowski \& Murdock, 1980; L. B. Ward, 1937). Pairwise $t$ tests, Bonferronicorrected for multiple comparisons, show that the primacy region for FR-constant does not differ significantly from that for FR-varied $[t(11)=0.6$, n.s. $]$ or SR $[t(11)=2.8$, n.s.]. The SR condition, however, does show significantly

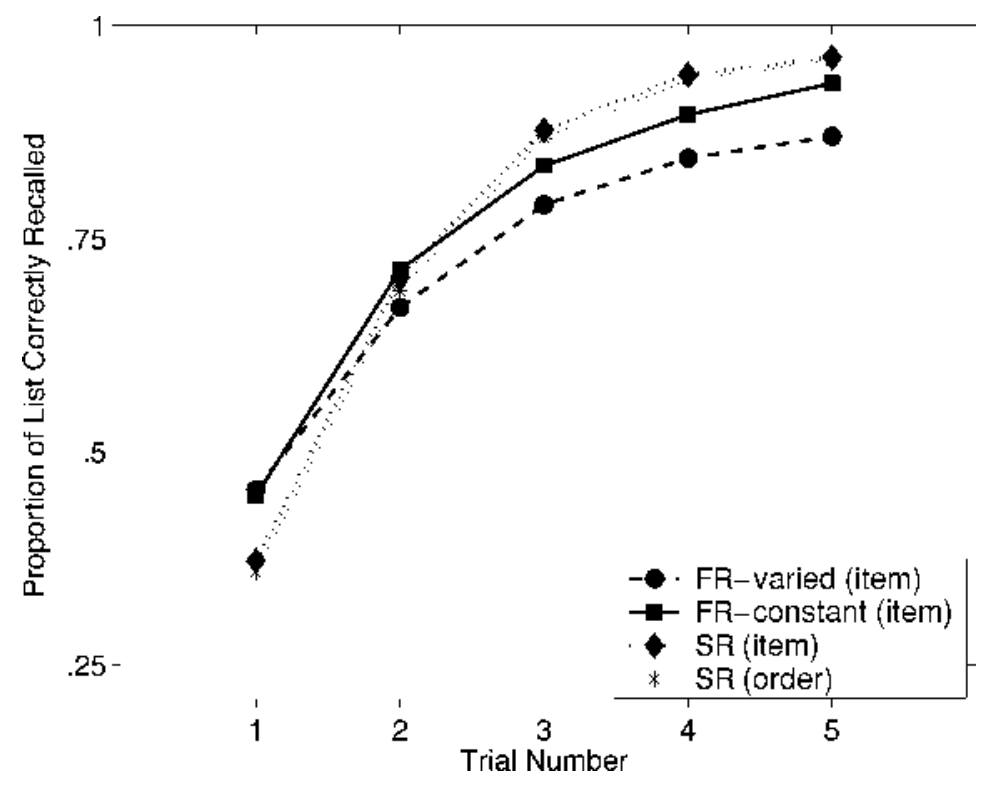

Figure 1. Learning curves. The four plotted curves show item scoring for free recall with varied presentation order (FR-varied [item]), free recall with constant presentation order (FR-constant [item]), and serial recall (SR [item]) conditions, and relative order scoring for serial recall (SR [order]). 


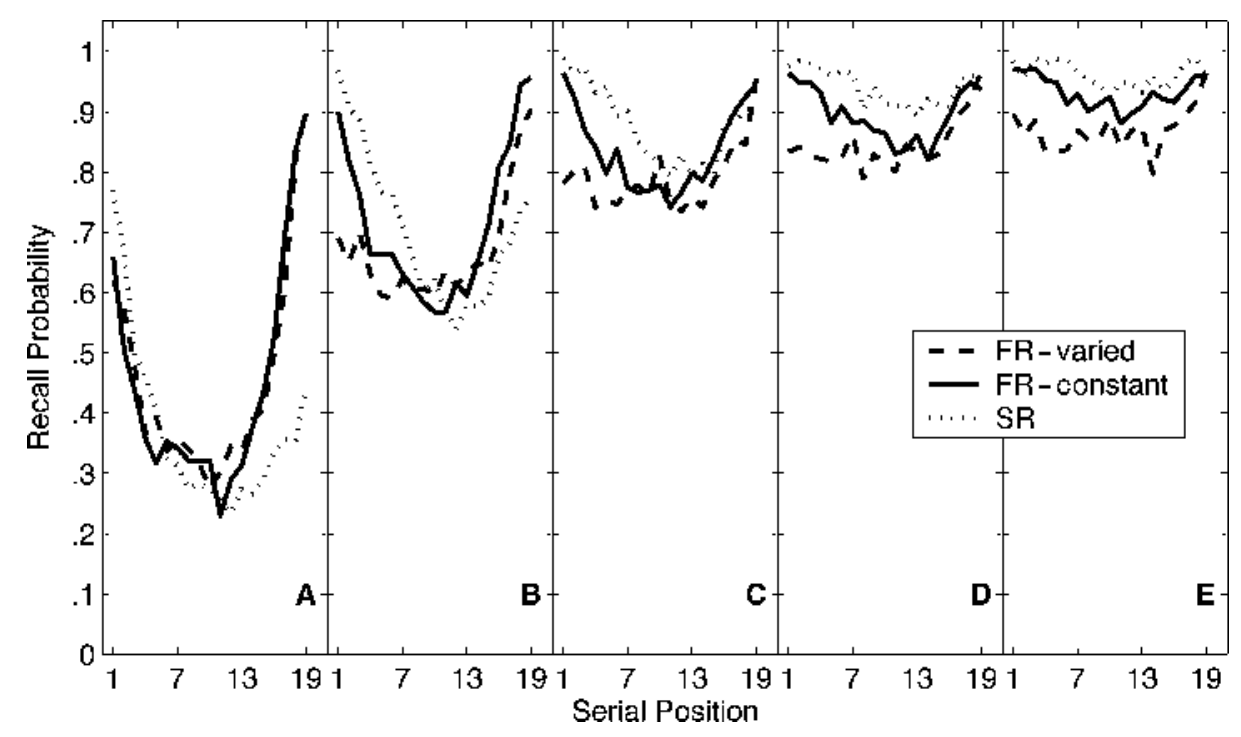

Figure 2. Serial position curves. (A) First-trial serial position curves for each of the three conditions: free recall with varied presentation order (FR-varied), free recall with constant presentation order (FR-constant), and serial recall (SR). (B) Second-trial serial position curves. (C) Third-trial serial position curves. (D) Fourth-trial serial position curves. (E) Fifth-trial serial position curves. In all cases, serial position curves are based on item scoring (see the text for details).

lower recall in the recency region than does either FRvaried $[t(11)=7.0, p<.001]$ or FR-constant $[t(11)=$ $6.2, p<.001]$, whereas FR-constant and FR-varied do not differ $[t(11)=1.1$, n.s. $]$. Thus, it seems that the participants approached first-trial free recall in the same manner for both FR-varied and FR-constant, regardless of their knowledge that word order in later trials would be varied or constant, respectively.

A significant interaction of condition, serial position bin, and trial $\left[F(16,176)=13.3, M S_{\mathrm{e}}=0.003, p<.001\right]$ indicates that the changes in the primacy and recency regions differ across conditions over the course of learning. Additional study-test trials serve to increase the overall level of the curves in all three recall conditions, but the similarity between FR-varied and FR-constant attenuates. Figure 2B shows that as early as Trial 2, the FR-constant curve exhibits a level of recall in the primacy region more similar to that seen in the SR curve than to that of the FR-varied curve. Bonferroni-corrected pairwise $t$ tests confirm that the Trial 2 primacy regions of the FR-constant and FR-varied conditions are significantly different $[t(11)=5.1, p<.001]$. The FR conditions diverge further as trials proceed, with the FRconstant condition looking more like the SR condition and less like the FR-varied condition.

\section{Conditional Response Probability}

Although learning curve and serial position curve analyses are useful in examining which items participants recall, they provide very little insight into how recall proceeds. Because it is reasonable to hypothesize that the specific trends in output order for a given listlearning paradigm could provide telling clues about the workings of memory mechanisms, newer methods of analysis have been developed. One such analysis is the conditional response probability as a function of lag (lag-CRP; Kahana, 1996). The lag-CRP measures the probability with which item $j$ is recalled after recall of item $i$, conditionalized on the availability of item $j$. These probabilities are plotted as a function of the lag $k$, where $k=j-i .{ }^{1}$ Take an example list ABSENCE HOLLOW PUPIL RIVER DARLING RAILWAY and an example recall trial RIVER DARLING ABSENCE. The transition from RIVER to DARLING is of lag $k=+1$, but lags of $k=-3$ (ABSENCE), $k=-2$ (HOLLOW), $k=-1$ (PUPIL), and $k=+2$ (RAILWAY) were also possible. The transition from DARLING to ABSENCE is of lag $k=-4$, but lags of $k=-3$ (HOLLOW), $k=-2$ (PUPIL), and $k=+1$ (RAILWAY) were also possible. Thus, the lag-CRP for this trial would have the values $P(k=$ $-4)=1 / 1=1.0, P(k=-3)=0 / 2=0, P(k=-2)=$ $0 / 2=0, P(k=-1)=0 / 1=0, P(k=+1)=1 / 2=.5$, and $P(k=+2)=0 / 1=0$. By plotting the probabilities that participants would make different kinds of temporal transitions during recall, Kahana found that transitions occur more frequently to items at nearby study positions than to items at distant study positions (termed associative contiguity) and that there is a forward asymmetry during recall.

Figures $3 \mathrm{~A}-3 \mathrm{C}$ show the lag-CRP functions for each recall condition over the course of learning. Since power functions have been found to describe lag-CRP curves (Kahana, Howard, Zaromb, \& Wingfield, 2002), functions 
of the form $\operatorname{CRP}(\operatorname{lag})=a|\operatorname{lag}|^{-b}$ were fit separately for positive and negative lags to each participant's data in each recall condition. The magnitude of the exponent, $b$, describes the degree to which the participants exhibit associative contiguity in recall transitions. Across-participants average exponents over Trials 1-5 for each of the three recall conditions appear in Figures 3D-3F. A 3 (condition) $\times 5$ (trial) $\times 2$ (transition direction) repeated measures ANOVA was computed on these exponents. A significant main effect of transition direction $[F(1,11)=$ $\left.165.16, M S_{\mathrm{e}}=0.28, p<.001\right]$ illustrates the forward asymmetry observed overall. In addition, a significant main effect of recall condition $\left[F(2,22)=50.82, M S_{\mathrm{e}}=\right.$ $1.48, p<.001]$ is moderated by a significant interaction of transition direction and recall condition $[F(2,22)=$ $\left.19.72, M S_{\mathrm{e}}=0.56, p<.001\right]$, which indicates that the degree of asymmetry differs across the three recall conditions. Further analysis reveals that this interaction is the result of significant simple effects of transition direction for the FR-constant $\left[F(1,11)=18.56, M S_{\mathrm{e}}=\right.$ $1.03, p<.005]$ and the $\operatorname{SR}\left[F(1,11)=55.34, M S_{\mathrm{e}}=\right.$ $0.69, p<.001]$ conditions, but not for the FR-varied condition $[F(1,11)=2.20$, n.s. $]$.

The changes in the lag-CRP over trials seen in Figures $3 \mathrm{~A}-3 \mathrm{C}$ for all three recall conditions are confirmed by a significant main effect of trial $[F(4,44)=16.01$, $\left.M S_{\mathrm{e}}=0.27, p<.001\right]$. Significant interactions of trial and transition direction $\left[F(4,44)=4.53, M S_{\mathrm{e}}=0.14\right.$, $p<.005]$ and trial and recall condition $[F(8,88)=32.73$, $\left.M S_{\mathrm{e}}=0.27, p<.001\right]$, as well as a marginally significant three-way interaction of trial, transition direction, and recall condition $\left[F(7,89)=2.15, M S_{\mathrm{e}}=0.22, p=\right.$ $.053]$ show that the changes in asymmetry over trials differ across the three recall conditions. Analysis of simple effects reveals that in Trial 1 (panels A1, B1, and C1 of Figure 3 ), the three conditions do not differ from one another $[F(2,22)=1.93$, n.s $]$, and all three conditions show a significant forward asymmetry $\left[F(1,11)=18.89, M S_{\mathrm{e}}=\right.$ $0.33, p<.005$ ], as is characteristic in free recall (Howard \& Kahana, 1999; Kahana, 1996; Kahana et al., 2002). Over trials, however, the three recall conditions show very different patterns of asymmetry, as is illustrated by Figures 3D-3F. As indicated by the significant interactions of trial and transition direction, as well as of trial and recall condition, the SR condition shows an increasing forward asymmetry over trials, the FR-constant condition shows a consistent level of forward asymmetry, and the FR-varied condition shows a decreasing forward asymmetry. In addition, the nonzero level of the average exponents in Figures $3 \mathrm{E}$ and $3 \mathrm{~F}$ shows that the FRconstant and SR conditions show associative contiguity across all trials, whereas Figure 3D shows that associative contiguity disappears over trials in the FR-varied condition. By Trial 5, virtually no associative contiguity is evident in the FR-varied condition (Figure 3, panel C5). In contrast, the exponents in the FR-constant condition remained at a constant level across trials (Figure 3E), and the SR condition forward exponent continued to increase over trials (Figure 3F). Although the degree of forward asymmetry differs between the FR-constant and the SR conditions, the transitions in the FR-constant condition resemble transitions in the SR condition con-
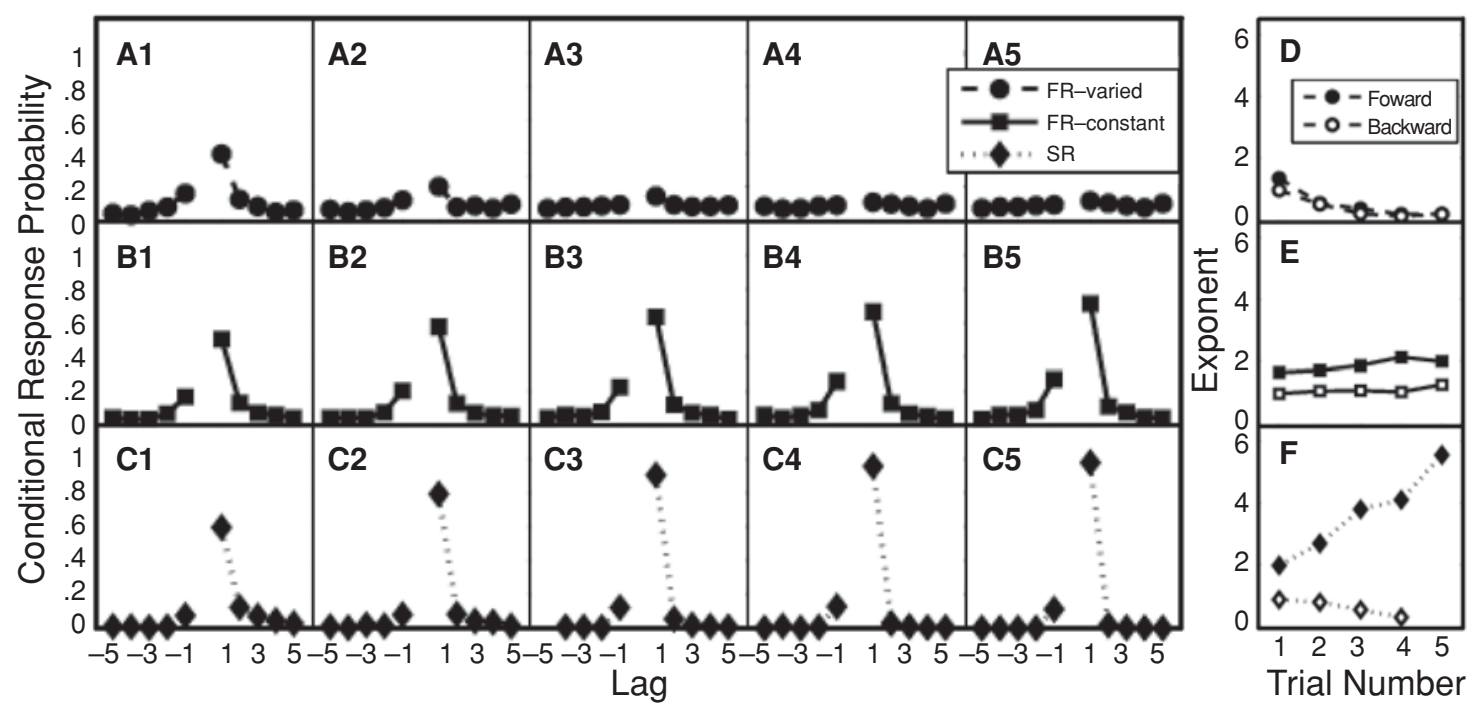

\footnotetext{
Figure 3. Associative contiguity effects. The left panels (A-C) show the conditional response probability as a function of lag (lag-CRP) across each of the five study-test trials (columns 1-5) and for each of the three conditions: A1-A5, free recall with varied presentation order (FR-varied); B1-B5, free recall with constant presentation order (FR-constant); C1-C5, serial recall (SR). To further quantify the changes in contiguity effects across trials and conditions, we fit the power function CRP(lag) $=a|\operatorname{lag}|^{-b}$ to individual participant lag-CRP functions. The rightmost panels (D-F) show the mean power function exponents, $b$, as a function of trial number for each of the three conditions: D, FR-varied; E, FR-constant; F, SR. Error bars represent $95 \%$ confidence intervals.
} 
siderably more than they resemble transitions in the FRvaried condition, which shows no associative contiguity and no forward bias.

\section{DISCUSSION}

We considered the relation between two classic learning paradigms: serial learning and multitrial free recall. In attempting to settle the question of whether the differences between these paradigms are due to recall instructions (free vs. serial) or item presentation order across trials (varied vs. constant), we compared three conditions: free recall with varied presentation order, free recall with constant presentation order, and serial recall. Specifically, the repeated contiguity between list items facilitates learning in the FR-constant condition beyond what is seen in traditional multitrial free recall. We find that temporal contiguity of study words across trials allows participants to more easily organize list items than when temporal contiguity is not present, leading to superior learning. Our results are consistent with the view that temporally defined interitem associations help to guide retrieval in all three recall conditions. The reason for the observed differences between the FRconstant and the FR-varied conditions is that in the FRconstant condition the associations learned on the previous presentations of the list reinforced those on the current presentation, whereas in the FR-varied condition they competed. In the SR condition, by requiring participants to recall the list in the order of study, one sees additional reinforcement of the same temporally defined associations during output. Thus, FR-varied, FR-constant, and SR form a continuum representing the degree to which contiguity at study and test plays a role in the associative structures formed in memory that ultimately guide recall.

On their first learning trial, the participants' performance was comparable to results from single-trial experiments in immediate free and serial recall. Both free recall conditions exhibited large recency effects and more moderate primacy effects, whereas in the SR condition, primacy was much larger than recency. The overall level of recall was higher in the free recall conditions than in serial recall, and analysis of item-to-item transitions revealed significant temporal contiguity effects in all conditions.

Considering that the participants were always aware of which condition they were being tested in, they could have easily adopted different Trial 1 strategies between the two FR conditions. However, it was not until later trials, when they could take advantage of the trial-to-trial temporal contiguity of list items provided in the FRconstant condition, that performance in the FR-constant condition began to diverge from performance in the FRvaried condition. Beginning in Trial 2 and continuing through Trial 5, FR-constant performance gradually took on similarities to performance in SR. In later trials, the shape of the FR-constant serial position curve strongly resembles the SR curve: Each exhibits nearly symmetrical primacy and recency effects, whereas the FR-varied serial position curve shows only recency.

Temporal contiguity effects, characterized by the lagCRP functions shown in Figure 3, decreased dramatically over trials in the FR-varied condition, remained constant over trials in the FR-constant condition, and increased asymmetrically in the SR condition. By Trial 5, temporal contiguity had virtually no effect on recall in the FR-varied condition, whereas in the SR condition, the participants made transitions almost exclusively to the next item in the presentation order. In the FR-constant condition, the participants used the constant temporal information to their advantage. They made transitions to nearby study positions from negative lags, as well as from positive lags, yielding a "loose" serial organization during recall.

These results follow from a straightforward associative account of list learning. In multitrial learning paradigms, participants have every reason to utilize associative information encoded on all previous trials, during both study and recall. When the presentation order is constant across trials, the current trial serves to reinforce previous trials. When the list order is shuffled on each trial, as in the FR-varied condition, item-to-item transitions reflect contributions of previous presentation orders and output orders in addition to that of the current presentation order. Thus, the resultant lag-CRP functions are flat when calculated using only the current presentation order.

Several factors may help to explain why Waugh (1961) did not detect a significant difference between learning curves in the FR-varied and the FR-constant conditions. First, her data set was very small: Nine participants each learned 6 lists in each condition. The present study included 12 participants, each of whom learned 21 experimental and 4 practice lists in each recall condition. Another drawback of Waugh's study was its lack of control in the SR condition: Although the participants were told to recall "early words before late ones," because the study used written recall, this instruction may not have always been followed by the participants, making it difficult to properly analyze errors in recall. The present study's use of recorded vocal recall prohibited the participants from returning to earlier serial positions later in the recall period. Finally, Waugh drew her conclusions entirely on the basis of learning curves, which provide an incomplete picture of the recall process. By analyzing recall transitions, the present study directly compared temporal contiguity effects in the three recall conditions.

The defining characteristic of the serial learning method is the requirement that participants recall list items in their presentation order. This is not so with free recall, the other principal method for studying list learning across multiple study-test trials. Our results suggest a common associative basis to both free and serial recall, with temporal contiguity effects being reinforced across multiple trials in serial recall but competing across trials 
in free recall. When presentation order is preserved across trials during free recall (as in our FR-constant condition), temporal contiguity is reinforced during the encoding phase, but the opportunity to recall items out of order often introduces competing associations. This condition thus reveals the distinct contributions of presentation order and recall instructions on the retrieval process.

\section{REFERENCES}

AdDis, K. M., \& Kahana, M. J. (2004). Decomposing serial learning: What is missing from the learning curve? Psychonomic Bulletin \& Review, 11, 118-124.

Anderson, J. R., Bothell, D., Lebiere, C., \& Matessa, M. (1998). An integrated theory of list memory. Journal of Memory \& Language, 38, 341-380.

BECKER, S., \& LiM, J. (2003). A computational model of prefrontal control in free recall: Strategic memory use in the California verbal learning task. Journal of Cognitive Neuroscience, 15, 821-832.

Botvinick, M., \& Plaut, D. C. (2004). Doing without schema hierarchies: A recurrent connectionist approach to routine sequential action and its pathologies. Psychological Review, 111, 395-425.

Brown, G. D. A., Preece, T., \& Hulme, C. (2000). Oscillator-based memory for serial order. Psychological Review, 107, 127-181.

Cowan, N., Baddeley, A. D., Elliott, E. M., \& Norris, J. (2003). List composition and the word length effect in immediate recall: A comparison of localist and globalist assumptions. Psychonomic Bulletin \& Review, 10, 74-79.

DALLETT, K. M. (1963). Practice effects in free and ordered recall. Journal of Experimental Psychology, 66, 65-71.

DrewnOwSKI, A., \& MuRdock, B. B. (1980). The role of auditory features in memory span for words. Journal of Experimental Psychology: Human Learning \& Memory, 6, 319-332.

FARrell, S., \& LeWANDOWSKY, S. (2002). An endogenous distributed model of ordering in serial recall. Psychonomic Bulletin \& Review, $\mathbf{9}$, 59-79.

Howard, M. W., \& Kahana, M. J. (1999). Contextual variability and serial position effects in free recall. Journal of Experimental Psychology: Learning, Memory, \& Cognition, 25, 923-941.

Howard, M. W., \& Kahana, M. J. (2002). A distributed representation of temporal context. Journal of Mathematical Psychology, 46, 269-299.

Hull, C. L., Hovland, C. I., Ross, R. T., Hall, M., Perkins, D. T., \& FiтcH, F. B. (1940). Mathematico-deductive theory of rote learning: A study in scientific methodology. New Haven, CT: Yale University Press.

Hulme, C., Stuart, G., Brown, G. D. A., \& Morin, C. (2003). Highand low-frequency words are recalled equally well in alternating lists: Evidence for associative effects in serial recall. Journal of Memory \& Language, 49, 500-518.

Johnson, G. J. (1991). A distinctiveness model of serial learning. Psychological Review, 98, 204-217.

Jung, J., \& SкеEbo, S. (1967). Multitrial free recall as a function of constant versus varied input orders and list length. Canadian Journal of Psychology, 21, 329-336.

Kahana, M. J. (1996). Associative retrieval processes in free recall. Memory \& Cognition, 24, 103-109.
Kahana, M. J., \& Caplan, J. B. (2002). Associative asymmetry in probed recall of serial lists. Memory \& Cognition, 30, 841-849.

Kahana, M. J., Howard, M. W., Zaromb, F., \& Wingfield, A. (2002). Age dissociates recency and lag recency effects in free recall. Journal of Experimental Psychology: Learning, Memory, \& Cognition, 28, 530-540.

Murdock, B. B. (1962). The serial position effect of free recall. Journal of Experimental Psychology, 64, 482-488.

Nairne, J. S., Neath, I., Serra, M., \& Byun, E. (1997). Positional distinctiveness and ratio rule in free recall. Journal of Memory \& Language, 37, 155-166.

RAAIJMAKERS, J. G. W. (2003). Spacing and repetition effects in human memory: Application of the SAM model. Cognitive Science, 27, 431452.

RASKIN, E., \& COOK, S. W. (1937). The strength and direction of associations formed in the learning of nonsense syllables. Journal of Experimental Psychology, 20, 381-395.

WARD, G., Woodward, G., Stevens, A., \& Stinson, C. (2003). Using overt rehearsals to explain word frequency effects in free recall. Journal of Experimental Psychology: Learning, Memory, \& Cognition, 29, 186-210.

Ward, L. B. (1937). Reminiscence and rote learning. Psychological Monographs, 49, 64.

WaUGH, N. C. (1961). Free versus serial recall. Journal of Experimental Psychology, 62, 496-502.

WILson, M. (1988). MRC Psycholinguistic Database: Machine-usable dictionary, version 2.00. Behavior Research Methods, Instruments, \& Computers, 20, 6-10.

\section{NOTE}

1. The lag-CRP estimates the probability of a transition to a given lag for successively recalled list items conditional on which list items are available for recall. Consider the recall of item $i$, followed by the recall of item $j$. The lag of the transition is calculated as $k=j-i$. A tally of such transitions accumulated over output positions measures the observed pattern of recall transitions as a function of lag. However, the availability of a transition of a given lag depends on the serial position of the current recalled item $i$. Transitions cannot extend beyond the boundaries of the list. Therefore, if the current recalled item is the last list item, a transition of lag $k=+1$ would not be possible. To correct for the difference in availabilities of various lags, we conditionalize this tally by dividing the number of observed transitions, $n(k)$, by the number of possible transitions, $d(k)$. In addition, because repetitions in recall are extremely rare, transitions to list items that have already been recalled on the current trial are not counted in either the numerator or the denominator. The lag-CRP is then calculated across lists for each participant as

$$
\mathrm{CRP}(k)=\frac{n(k)}{d(k)} .
$$

Unlike Kahana (1996), we collapse across all output positions. We present lag-CRPs that represent the across-participants average values.

(Manuscript received February 10, 2004; revision accepted for publication August 17, 2004.) 